American Journal of Applied Sciences 6 (3): 430-438, 2009

ISSN 1546-9239

(C) 2009 Science Publications

\title{
Determination of Nonlinear Optimal Feedback Law for Satellite Injection Problem Using Neighboring Optimal Control
}

\author{
${ }^{1}$ Hamed Hossein Afshari, ${ }^{1}$ Alireza Basohbat Novinzadeh, ${ }^{1}$ Jafar Roshanian \\ ${ }^{1}$ Department of Aerospace Engineering, \\ K. N. Toosi University of Technology, P.O Box 16765-3381, \\ Tehran, Iran
}

\begin{abstract}
An optimal trajectory design of a nonlinear satellite injection problem for transfer to a final target orbit by minimizing the time was investigated. Indeed, this design was an exact solution to the nonlinear two-point boundary value problem which determined optimal control history as well as optimal state trajectories in the open-loop form. Furthermore, the obtained optimal guidance strategy was exerted in the closed-loop form against the environment disturbances using neighboring optimal control method in the exact solution. Neighboring Optimal Control (NOC) law could produce timevariant feedback gains minimizing the performance measure to second order for perturbations from a nominal optimal path. Generally, this law was a function of perturbations appeared in the states and constraints and could be computed utilizing the backward sweep method. The simulation results indicated that the presented methodology was successfully utilized in the real world applications with good robustness to each noise or disturbance produced in each state variable.
\end{abstract}

Keywords: Satellite Injection, Closed-loop Guidance, Exact Solution

\section{INTRODUCTION}

Determination of closed-loop feedback control law is not usually feasible for many nonlinear dynamic systems. In addition, there exist certain difficulties associated with the numerical determination of openloop optimal control solution for nonlinear systems, such as slow convergence rate and high sensitivity to initial guesstimates. Besides, if one manages to overcome these inherent difficulties, the determined optimal control strategy will be in an open-loop form, and thus fully dependent on the initial condition. Obviously, perturbation and noise process will make the optimal trajectory deviate from its ideal predicted values in any actual operating environment. The task of controller design for nonlinear systems which requires minimization of a functional in addition to simultaneous satisfaction of physical constraints is usually formidable and often lead to sub-optimal solutions. In general, optimal formulations of nonlinear dynamic systems either through dynamic programming or variational approach lead to nonlinear partial differential equations. Numerical solution of these equations when dealing with complex nonlinear systems is always difficult, especially for real world physical problems. Obtaining closed-loop control laws, intensifies the inherent difficulty involved and is only exceptionally determined for some rare cases.

It should be considered that optimal control solution of a dynamic system can be classified into two main categories of open-loop and closed-loop. Open-loop optimal controls are only functions of time and once the system starts from a known initial condition, the predetermined optimal control activate to take the trajectory toward the final conditions with no feedback of states along the path. It is a task that will perform the job well if there is small perturbation or small noise in the state variables and in the terminal conditions. On the other hand, closed-loop optimal controls are functions of time and states and in essence posses a sort of inherent robustness against noise and undesirable disturbance presented in any actual operating environment. It should be noted that closed-loop optimal control solutions are seldom possible for nonlinear dynamic systems, even though they are highly desirable for their robust characteristics. However, open-loop control laws are highly sensitive with respect to noise and external disturbances and therefore are not much preferred for real world applications.

Corresponding Author: Hamed Hossein Afshari, Department of Aerospace Engineering, K. N. Toosi University of Technology, P.O Box 16765-3381, Tehran, Iran, Tel: +98-9366691025 
It should be noted that the technique of NOC is a continuous linear feedback law can produce timevariant feedback gains which minimize the performance index ${ }^{[1]}$. This law is established on the linear perturbation equations around the nominal optimum path where the weighting factors in the quadratic performance index are the second partial derivatives of the variational Hamiltonian. Open-loop optimal control solution for nonlinear launching in the gravity of moon was obtained either by dynamic programming approach or through a variational formulation of the optimal control problem. Also, the neighboring extremals are given for injection into orbit ${ }^{[1]}$. Also, time-optimal solution of nonlinear landing mission in polar coordination system was found utilizing a numerical technique named linear programming. This law is an exact solution to the two-point boundary value problem associated with the first variation necessary conditions $^{[2]}$. The results of minimum-time Feedback Laws $^{[3]}$ are used to validate the results of an analytical open-loop strategy proposed in this paper. Jardin and Bryson used the technique of NOC to develop an algorithm for optimizing aircraft trajectories in general wind fields computing time-varying linear feedback gains $^{[4]}$. Furthermore, Pourtakdoust and Novinzadeh presented a time-optimal open-loop strategy for nonlinear lunar landing mission obtained using an analytical technique. Then in order to create closedloop fuzzy guidance logic, a fuzzy algorithm was augmented to the variational function of the problem ${ }^{[6]}$. More recently, Palma has worked on optimal predictive control by discretizing nonlinear dynamics systems ${ }^{[8]}$. Also, Novinzadeh presented a close form solution of the generated optimal formulation of mass-variant module utilizing the regular perturbation theory and applied fuzzy logic to obtain closed-loop guidance strategy ${ }^{[9]}$. An optimal guidance law that minimized the commanded acceleration in three dimensions was obtained by Souza ${ }^{[10]}$. Feeley and Speyer used a technique to develop a real-time guidance scheme for the Advanced Launch System. Their approach is to construct an optimal guidance law based upon an asymptotic expansion dealing with regular perturbation theorem $^{[11]}$. In this way, Leung and Calise proposed a hybrid approach to launch vehicle guidance for ascent to orbit injection. The feedback guidance approach is based on a piecewise nearly analytical solution evaluating using a collocation method ${ }^{[12]}$. Dabbous and Ahmed presented their work on nonlinear optimal regulator problem for final time unspecified systems ${ }^{[13]}$. There are many studies performed by Nuidiu on the closed-loop optimal control using singular perturbation methods ${ }^{[14,15]}$. In addition, Bei applied the line-of-sight homing technology to achieve more precision lunar landing. Hardware realization concept of precise landing mission was also simply discussed ${ }^{[16]}$. Suzuki and Yoshizawa introduced a new guidance law for lunar landing module by minimizing fuel expenditure considered as a reference for verifying the results of presented work $^{[17]}$. Also, Naghash and Esmaelzadeh developed an explicit guidance law that maximized terminal velocity for a reentry vehicle to a fixed target. Acceleration commands were derived by solving an inverse problem related to Bezier parameters and an optimal Bezier curve was determined by solving a genetic algorithm $^{[18]}$.

The presented study concentrates on finding an analytical methodology to compute the open-loop timeoptimal guidance strategy for satellite injection problem. In addition, a closed-loop control law for protecting the launched satellite on the obtained trajectory is determined against small perturbations using NOC method in the exact form. The mentioned perturbations applied on the state variables of injection dynamic system can be appeared in the real world application such as measurement system performance.

\section{MATERIALS AND METHODS}

The main results of ${ }^{[1]}$ which presents a detail explanation about NOC theorem and its application in the optimal control problems are summarized in the following. At first, consider that the optimal control problem of dynamic systems can be formulated using calculus of variations. In this way, one usually assumes a mathematical representation of the current system as a first order differential equation

$\dot{\vec{x}}=f(\vec{x}(t), \vec{u}(t), t), \quad t_{0} \leq t \leq t_{f}$

where $\vec{x}(t)$ and $\vec{u}(t)$ are n-dimensional state vector and m-dimensional control vector, respectively. A conventional form of performance measure can be expressed as:

$J=\Phi\left[\vec{x}\left(t_{f}\right), t_{f}\right]+\int_{t_{0}}^{t_{f}} L[\vec{x}(t), \vec{u}(t), t] d t$

where $\Phi\left[\vec{x}\left(t_{f}\right), t_{f}\right]$ is the penalty function for the final states at the final time. The first step toward a variational method is to find the system Hamiltonian as:

$H=L[\vec{x}(t), \vec{u}(t), t]+p^{T} f(\vec{x}(t), \vec{u}(t), t)$

By considering the terminal constraints as:

$\Phi=\varphi+v^{T} \vec{\psi}$ 
where $\vec{\psi}$ is a q-dimensional vector named constraint function and $\varphi$ is a constant terminal constraint. Since the final time is free, so that the necessary conditions for optimality are derived as:

$$
\begin{aligned}
& \dot{p}=-H_{x} ; \quad p\left(t_{f}\right)=\left.\Phi_{x}\right|_{t_{f}} \\
& H_{u}=p^{T} f_{u} ; \quad H\left(t_{f}\right)=-\Phi_{t} \mid t_{f}
\end{aligned}
$$

Now, assume small perturbations from the obtained extermal path produced by small perturbations in the initial state $\delta \vec{x}\left(t_{0}\right)$ and in the terminal condition $\delta \vec{\psi}$. This expected that if the final time is specified, small perturbations such as $\delta \vec{x}(t), \delta p(t), \delta \vec{u}(t)$ and $d \vec{v}$ will be appeared as:

$\delta p=S(t) \delta \vec{x}(t)+R(t) d \vec{v}$

$\delta \vec{\psi}=R^{T}(t) \delta \vec{x}(t)+Q(t) d \vec{v}$

where $d \vec{v}$ and $\delta \vec{\psi}$ are infinitesimal vectors. These matrices can be determined by solving the following differential equations with regarding its augmented boundary conditions by utilizing backward sweep method:

$$
\begin{aligned}
& \dot{S}=-S A-A^{T} S+S B S-C \\
& S\left(t_{f}\right)=\left[\varphi_{x x}+v^{T} \vec{\psi}_{x x}\right]_{t=t_{f}}, \\
& \dot{R}=-\left(A^{T}-S B\right) R, R\left(t_{f}\right)=\left[\vec{\psi}_{x}^{T}\right]_{t=t_{f}}, \\
& \dot{Q}=R^{T} B R, Q\left(t_{f}\right)=R^{T} B R
\end{aligned}
$$

It can be easily shown that equation 7 forms a matrix Riccati equation due to contain a quadratic term in S. In this way, the matrices $\mathrm{A}, \mathrm{B}$ and $\mathrm{C}$ can be formulated as:

$$
\begin{aligned}
& A(t)=f_{x}-f_{u} H_{u u}^{-1} H_{u x}, \\
& B(t)=f_{u} H_{u u}^{-1} f_{u}^{T}, \\
& C(t)=H_{x x}-H_{x u} H_{u u}^{-1} H_{u x}
\end{aligned}
$$

Finally, by using previous equation sets, the NOC law is abbreviated as:

$$
\begin{aligned}
\delta \vec{u}(t)= & -\left\{\left[f_{u}^{T}\left(S-R Q^{-1} R^{T}\right)+H_{u x}\right] \delta \vec{x}\right. \\
& \left.+f_{u}^{T} R Q^{-1} \delta \vec{\psi}\right\} H_{u u}^{-1} \rightarrow \\
\delta \vec{u}(t)= & -\Lambda_{1}(t) \delta \vec{x}-\Lambda_{2}(t) \delta \vec{\psi}
\end{aligned}
$$

The NOC law when the final time unspecified was obtained in equation 11. Besides, if the final time is unspecified, this law will be formulated in the similar form. But note that small perturbation arises in the final time which discussed in ${ }^{[1]}$ perfectly.

\section{ANALYTICAL OPEN-LOOP SOLUTION TO THE SATELLITE INJECTION PROBLEM}

Consider an idealized point-mass satellite at the origin of inertial frame $(x, y)$ at $t=0$, moving under the action of a constant propulsive force making a control angle $\beta(t)$ with the horizon. Obviously, the position and velocity vector of the vehicle will change due to the action of forces acting on it. The problem is to determine the time-optimal control strategy of this system for transfer to a final target orbit. Based on Fig. 1 , the governing state-space equations are:

$\left\{\begin{array}{l}\frac{d u}{d t}=\frac{T}{m} \cos \beta, \\ \frac{d v}{d t}=\frac{T}{m} \sin \beta-\frac{g}{m}, \\ \frac{d y}{d t}=v\end{array}\right.$

with its appropriate boundary conditions:

$u(t=0)=0, v(t=0)=0, y(t=0)=0$,

$u\left(t=t_{f}\right)=U_{f}, v\left(t=t_{f}\right)=0, y\left(t=t_{f}\right)=h$

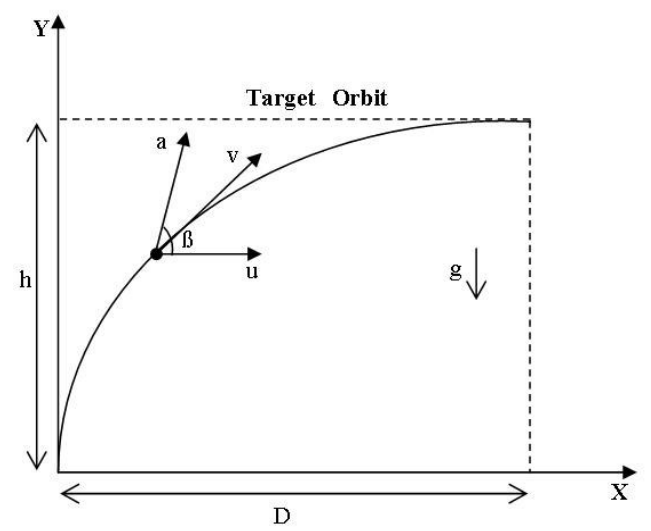

Fig. 1: Geometry of the satellite injection mission

For a better physical understanding and reaching an analytical explicit solution, the governing equations and the associated boundary conditions are nondimensionalized using a set of assumed reference parameters $\left(u *, v *, u^{*}\right)$ :

$\bar{u}=\frac{u}{U *}, \bar{v}=\frac{v}{U *}, \bar{y}=\frac{y}{y *}$,

$\tau=\frac{t}{t *}, \frac{d}{d t}=\frac{1}{t *} \frac{d}{d \tau}$

for time-optimal problems where $t_{f}$ is free, one usually utilizes the final time as a referencing condition for 
determining non-dimensional parameters. Also, the reference parameters are:

$U *=U_{f}, y *=h, t^{*}=\frac{h}{U_{f}}$

Now, by using the above non-dimensional state variable equations, the transformed equations become:

$\left\{\begin{array}{l}\frac{d \bar{u}}{d \tau}=w_{1} \cos \beta, \\ \frac{d \bar{\nu}}{d \tau}=w_{1} \sin \beta-w_{2}, \\ \frac{d \bar{y}}{d \tau}=w_{3} \bar{v}\end{array}\right.$

where

$w_{1}=\frac{T t^{*}}{m U *}, w_{2}=\frac{g t *}{m U *}, w_{3}=\frac{U * t *}{y *}$

with non-dimensional boundary conditions:

$\bar{u}(\tau=0)=0, \bar{v}(\tau=0)=0, \bar{y}(\tau=0)=0$,

$\bar{u}\left(\tau=\tau_{f}\right)=1, \bar{v}\left(\tau=\tau_{f}\right)=0, \bar{y}\left(\tau=\tau_{f}\right)=1$

Since the problem is determining the control action of $\beta=\beta(t)$ required for time-optimal control policy for transferring the satellite to the specified orbit, so that the performance measure is defined as:

$J=\int_{0}^{\tau_{f}} d \tau$

and the corresponding Hamiltonian based on equation 3 will be determined as:

$H=1+p_{1} w_{1} \cos \beta+p_{2}\left(w_{1} \sin \beta-w_{2}\right)+p_{3} w_{3} \bar{v}$

Using the co-state equations and optimality relation, one can find the following relations for optimal control and co-state parameters:

$$
\begin{gathered}
\tan \beta=\frac{p_{2}}{p_{1}} \\
p_{1}=k_{1}, \\
p_{2}=-k_{3} w_{3} \tau+k_{2}, \\
p_{3}=k_{3}
\end{gathered}
$$

where $k_{1}, k_{2}$ and $k_{3}$ are constant parameters which should be determined. Now, by using the above relations, one can find an implicit formulation for the optimal control as:

$\tan \beta=-\frac{k_{3} w_{3}}{k_{1}} \tau+\frac{k_{2}}{k_{1}}$

Now, with respect to equation 23 and existing relationship between $\beta$ and $\tau$, the time derivatives appeared in the state-space equations can be written with respect to $\beta$. By using this technique, $\beta$ now becomes independent variable and therefore, the transformed state-space equations can be rewritten as:

$$
\left\{\begin{array}{l}
\frac{d \bar{u}}{d \beta}=\frac{k_{1} w_{1}}{k_{3} \cos \beta}, \\
\frac{d \bar{\nu}}{d \beta}=\frac{k_{1} w_{1} \sin \beta-k_{1} w_{2}}{k_{3} \cos \beta}, \\
\frac{d \bar{y}}{d \beta}=\frac{k_{1} w_{3} \bar{v}}{k_{3} \cos ^{2} \beta}
\end{array}\right.
$$

In addition, non-dimensional initial conditions and terminal conditions are defined as:

$u\left(\beta_{0}\right)=0, v\left(\beta_{0}\right)=0, y\left(\beta_{0}\right)=0$,
$u\left(\beta_{f}\right)=1, v\left(\beta_{f}\right)=0, y\left(\beta_{f}\right)=1$.

for a set of assumed values of the parameters $\left(U_{0}, h, T, m, g\right)$ the geometrical parameters $\left(w_{1}, w_{2}, w_{3}\right)$ can be determined. For example if $U_{0}=h=T=m=1$ and $g=1 / 3$, the geometrical parameters are $w_{1}=1, w_{2}=1 / 3, w_{3}=1$.

Considering the simpler form of equation 24 and applying initial conditions, it is integrated to yield the result as a function of the control angle $\beta$ in the following formulation:

$$
\begin{aligned}
\bar{u}(\beta)= & \frac{k_{1}}{k_{3}} \ln \frac{(\sec \beta+\tan \beta)}{\left(\sec \beta_{0}+\tan \beta_{0}\right)} \\
\bar{v}(\beta)= & \frac{-\frac{k_{1}}{3} \sin \left(\beta-\beta_{0}\right)-k_{1}\left(\cos \beta-\cos \beta_{0}\right)}{k_{3} \cos \beta \cos \beta_{0}} \\
\bar{y}(\beta)= & \frac{k_{1}^{2}}{6 k_{3}^{2}}\left[3 \ln \frac{\sec \beta+\tan \beta}{\sec \beta_{0}+\tan \beta_{0}}+3 \tan \beta \times\right. \\
& \left(\frac{1}{\cos \beta}-\frac{2}{\cos \beta_{0}}\right)-\left(\frac{1}{\cos ^{2} \beta}-\frac{1}{\cos ^{2} \beta_{0}}\right) \\
& \left.-2 \tan \beta_{0}\left(\tan \beta-\tan \beta_{0}\right)-3 \frac{\sin _{0}}{\cos ^{2} \beta_{0}}\right]
\end{aligned}
$$

Obviously for explicit results, it is necessary to specify the values of $k_{1}, k_{2}, k_{3}, \beta_{0}, \beta_{f}$ and $\tau_{f}$. This can be accomplished by using the six boundary conditions presented as:

$\bar{u}\left(\beta_{f}\right)=1, \bar{v}\left(\beta_{f}\right)=0, \bar{y}\left(\beta_{f}\right)=1$,
$H\left(\beta_{f}\right)=0, \beta\left(\tau_{0}\right)=\beta_{0}, \beta\left(\tau_{f}\right)=\beta_{f}$

In this way, the following algebraic equations are derived as:

$k_{2} \cos \beta_{0}-k_{1} \sin \beta_{0}=0$,

$\left(k_{2}-k_{3} \tau_{f}\right) \cos \beta_{f}-k_{1} \sin \beta_{f}=0$, 
$1+k_{1} \cos \left(\beta_{f}\right)+\left(k_{2}-k_{3} \tau\right)\left(\sin \left(\beta_{f}\right)-1 / 3\right)=0$,

$\frac{k_{3}}{k_{1}}+\ln \left(\sec \left(\beta_{f}\right)+\tan \left(\beta_{f}\right)\right)$

$-\ln \left(\sec \left(\beta_{0}\right)+\tan \left(\beta_{0}\right)\right)=0$,

$\frac{1}{\cos \beta_{0}}-\frac{\tan \beta_{0}}{3}-\frac{1}{\cos \beta_{f}}+\frac{\tan \beta_{f}}{3}=0$,

$\frac{1}{2}\left(\frac{\sin \beta_{f}}{\cos ^{2} \beta_{f}}-\frac{\sin \beta_{0}}{\cos ^{2} \beta_{0}}\right)+\frac{1}{2} \ln \left(\frac{\sec \beta_{f}+\tan \beta_{f}}{\sec \beta_{0}+\tan \beta_{0}}\right)$

$-\frac{\sin \beta_{f}}{\cos \beta_{0} \cos \beta_{f}}-\frac{1}{6}\left(\frac{1}{\cos ^{2} \beta_{f}}-\frac{1}{\cos ^{2} \beta_{0}}\right)$

$+\frac{\tan \beta_{f} \tan \beta_{0}-\tan ^{2} \beta_{0}}{3}-\left(\frac{k_{3}}{k_{1}}\right)^{2}=0$

By solving the above algebraic equations, the six unknown parameters can be easily found as:

$\tau_{f}=2.213, \beta_{0}=1.428 \mathrm{rad}, \beta_{f}=-1.275 \mathrm{rad}$,

$k_{1}=0.221, k_{2}=0.725, k_{3}=0.995$

Consequently, the optimal control histories and optimal state trajectories are determined as:

$\beta=\tan ^{-1}(6.671-4.499 \tau)$,

$\bar{u}(\beta)=0.577-0.222 \ln (\sec \beta+\tan \beta)$,

$\bar{v}(\beta)=0.0741 \frac{-13.57 \cos \beta-\sin \beta+3}{\cos \beta}$,

$\bar{y}(\beta)=-0.223 \tan \beta+0.025 \ln (\sec \beta+\tan \beta)$

$$
+0.025 \tan \beta \sec \beta-0.008 \sec ^{2} \beta+0.689
$$

It should be noted that the investigated results are identical with the analytical results of reference [3]. By regarding this fact that the solution parameters $\bar{u}, \bar{v}, \bar{y}$ and $\bar{x}$ have been expressed in the terms of $\beta$, and $\beta$ is related to $\tau$, so that the solution parameters could be expressed respect to $\tau$. As a better demonstration, the optimal results are compared by using four satellite injection scenarios with different initial conditions tabulated in the table 1. Also, the variation of initial and final thrust angle are visible.

Table 1: Comparison between Four Satellite Injection Scenarios with Different Initial Conditions

\begin{tabular}{ccccc}
\hline$\left(\mathrm{h}_{0}, \mathrm{U}_{0}\right)$ & $(1,1)$ & $(1,0.75)$ & $(0.75,1)$ & $(0.75,0.75)$ \\
\hline $\mathrm{k}_{1}:$ & -0.2211 & -0.1436 & -0.2755 & -0.1779 \\
$\mathrm{k}_{2}:$ & -1.4753 & -1.4896 & -1.4614 & -1.4841 \\
$\mathrm{k}_{3}:$ & -0.9951 & -1.0292 & -1.1152 & -1.1724 \\
$\tau_{\mathrm{f}}:$ & 2.2114 & 2.1661 & 1.9483 & 1.8920 \\
$\beta_{0}:$ & 1.4220 & 1.4747 & 1.3845 & 1.4515 \\
$\beta_{\mathrm{f}}:$ & -1.2748 & -1.3791 & -1.2013 & -1.3331 \\
\hline
\end{tabular}

The computed control policy is in the open-loop form and in order to protecting the moving satellite against the environment disturbances, the closed-loop control law must be determined. For achieving to this purpose, the NOC method is utilized.

\section{APPLICATION OF NOC LAW FOR CLOSED- LOOP SOLUTION}

In this stage, the optimal closed-loop solutions are determined analytically using the non-dimensional form of NOC law. At first, the unknown matrixes namely $A(\tau), B(\tau)$ and $C(\tau)$ are determined using the equation 10 as following:

$$
\begin{aligned}
& A(\tau)=\left[\begin{array}{lll}
0 & 0 & 0 \\
0 & 0 & 0 \\
0 & 1 & 0
\end{array}\right], C(\tau)=0_{3 \times 3}, \\
& B(\tau)=4.523 \mathrm{c}(\beta)\left[\begin{array}{ccc}
\mathrm{s}^{2}(\beta) & -\mathrm{s}(\beta) \mathrm{c}(\beta) & 0 \\
-\mathrm{s}(\beta) \mathrm{c}(\beta) & \mathrm{c}^{2}(\beta) & 0 \\
0 & 0 & 0
\end{array}\right]
\end{aligned}
$$

Computing $A(\tau), B(\tau)$ and $C(\tau)$, it is possible to determine $R(\tau)$ by solving the differential equation contained in equation 8 as follows:

$\frac{d R_{3 \times 3}}{d \tau}=\left[\begin{array}{ccc}0 & 0 & 0 \\ -R_{31} & -R_{32} & -R_{33} \\ 0 & 0 & 0\end{array}\right], R\left(\tau_{f}\right)=I_{3 \times 3}$

Each member of above matrix equation forms a differential equation which must be integrated backward from the terminal time to the current time. Furthermore, by considering equation 14, it can find that the thrust angle $\beta(\tau)$ is related to time, thus $Q(\tau)$ could be found by solving a set of independent differential equations with corresponding boundary condition in the following form:

$$
\begin{aligned}
& \frac{d Q_{3 \times 3}}{d \tau}=4.523 \mathrm{c}(\beta) \times \\
& {\left[\begin{array}{ccc}
\mathrm{s}^{2}(\beta) & -\mathrm{s}(\beta) \mathrm{c}(\beta) & -\mathrm{s}(\beta)\left(\tau_{f}-\tau\right) \mathrm{c}(\beta) \\
-\mathrm{s}(\beta) \mathrm{c}(\beta) & \mathrm{c}^{2}(\beta) & \left(\tau_{f}-\tau\right) \mathrm{c}^{2}(\beta) \\
-\mathrm{s}(\beta) \mathrm{c}(\beta)\left(\tau_{f}-\tau\right) & \mathrm{c}^{2}(\beta)\left(\tau_{f}-\tau\right) & \left(\tau_{f}-\tau\right)^{2} \mathrm{c}^{2}(\beta)
\end{array}\right],}
\end{aligned}
$$

$Q\left(\tau_{f}\right)=\varnothing_{3 \times 3}$

Therefore, $Q(\tau)$ is obtained by backward integration from each member of the above matrix differential equation with respect to the time analytically. In effect, the differential equations are swept backward from the terminal condition to the current condition in the exact solution. 


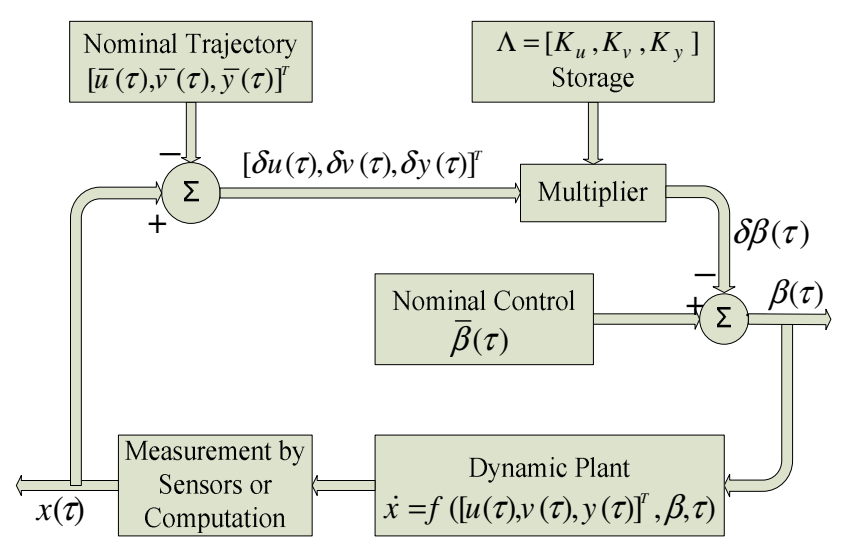

Fig. 2: Block-diagram of NOC law for determining closedloop trajectory for satellite injection mission.

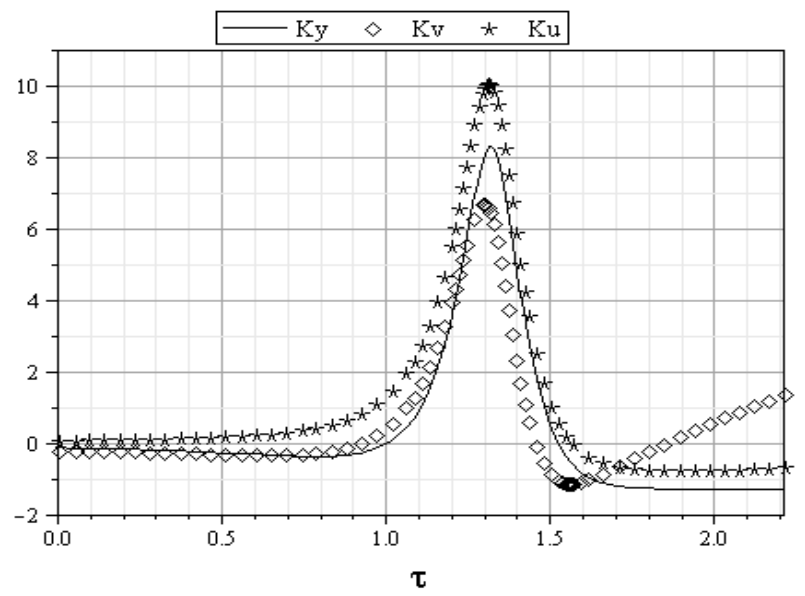

Fig. 3: Neighboring optimum gains for satellite injection mission.

According to equation 7, since the co-state equations are not functions of state variables, so that the matrix $S(\tau)$ are equal to zero and solving the Riccati equation is not required. Note that because the satellite injection problem is in the class of a terminal guidance problem, thus the terminal constraints perturbation will be equal to zero. As result of the mentioned reason, the NOC law is a function of state variables perturbation only. The NOC block-diagram for determining optimal closedloop control law for satellite injection problem is depicted in Fig. 2. Also, by computing the time perturbation, it is found that it is less than $10^{-3}$, so that it is negligible. By regarding the previous relations, the NOC law could be found in the following formulation:

$$
\delta \beta(\tau)=K_{u}(\tau) \delta u(\tau)+K_{v}(\tau) \delta v(\tau)+K_{y}(\tau) \delta y(\tau)
$$

where the perturbed terms, $\delta u(\tau), \delta v(\tau)$ and $\delta y(\tau)$ are small perturbations applied to the state variables of satellite injection system. Also, $K_{u}(\tau), K_{v}(\tau)$ and $K_{y}(\tau)$ are neighboring optimum gains for determination of optimal closed-loop guidance law.

\section{RESULTS AND DISCUSSION}

The time histories of neighboring optimal feedback gains for satellite injection problem are depicted in Fig. 3. Having obtained these gains, the time optimal closedloop control solution can be computed by using equation 44. By observing the figures, it can be easily shown that the NOC law can satisfy each of the boundary condition of satellite injection problem by minimizing the time introduced as the performance measure. Obviously, the position vector components $x(\tau)$ and $y(\tau)$ are computed by integrating from velocity vector components $u(\tau)$ and $v(\tau)$ with respect to the time, respectively.

There are several source of disturbance in the operating environment which could effect on the performance of our determined optimal feedback law. To investigate robustness potentials of the proposed NOC, the satellite injection system is analyzed under the influence of small disturbances exerted in the state feedback (measurement unit) of the satellite injection dynamical. These state disturbances modeled similar to the control actuation noise are taken as:

$\xi(t)=\varepsilon \sin (\omega t)$

$\bar{u}_{\xi}=\bar{u}+\xi(t), \bar{v}_{\xi}=\bar{v}+\xi(t)$

With the following set of parameters $\omega_{1}=250$ and $\varepsilon=0.01$. The perturbed thrust angle for protecting the launched satellite against the environment disturbances is depicted in Fig. 4. In addition, the comparisons between open-loop and closed-loop optimal results are illustrated in Fig. 5, Fig. 6, Fig. 7, Fig. 8 and Fig. 9. Also as can be seen in Fig .10 and Fig. 11, there exists an excellent agreement between open-loop and closedloop solution in the state-space trajectories. One can easily verify from the results that the measurement disturbance has no effect on the performance of NOC, while the system behaves in an oscillatory fashion when it is not in the closed-loop form with NOC. The simulation results indicate that the feedback performance is in close agreement with the exact solution and thus, the presented methodology can be successfully utilized in the real world application with good robustness to each noise or disturbance produced in the each state variable. 


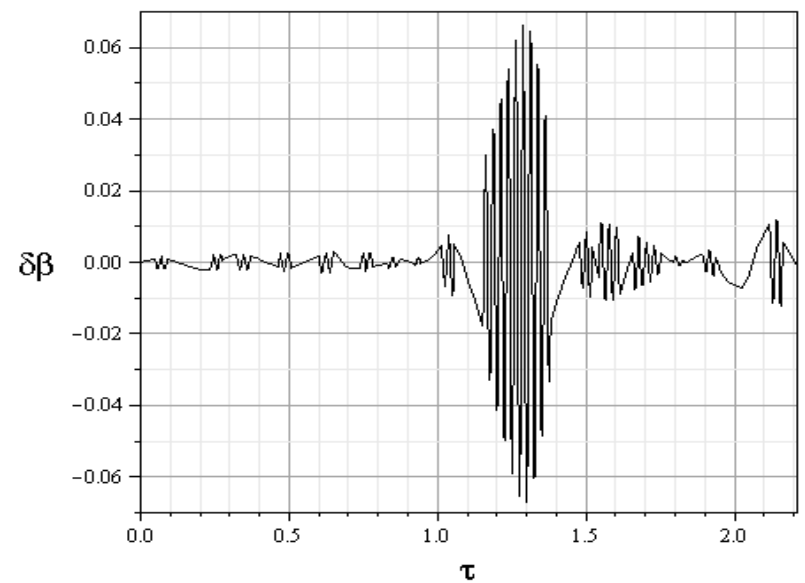

Fig. 4: The perturbed thrust angle due to apply disturbance obtained using NOC law.

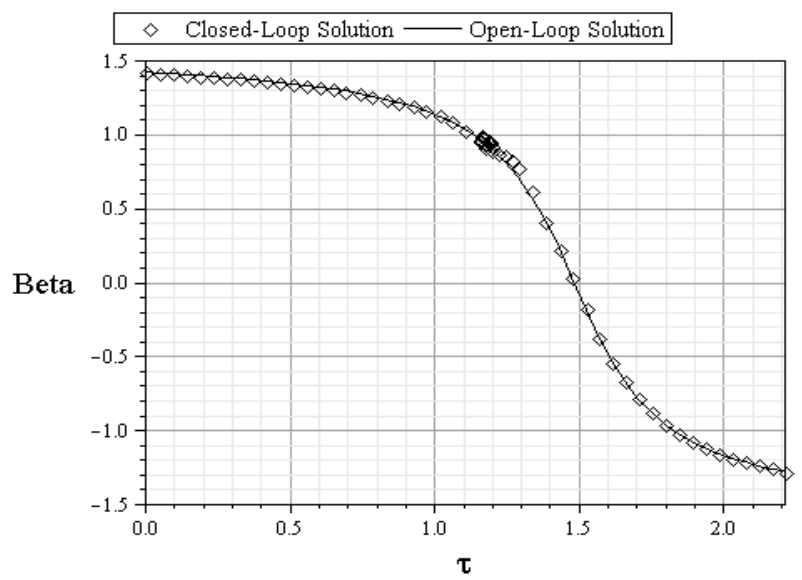

Fig. 5: Comparison between open-loop and closed-loop solution of time-optimal thrust angle.

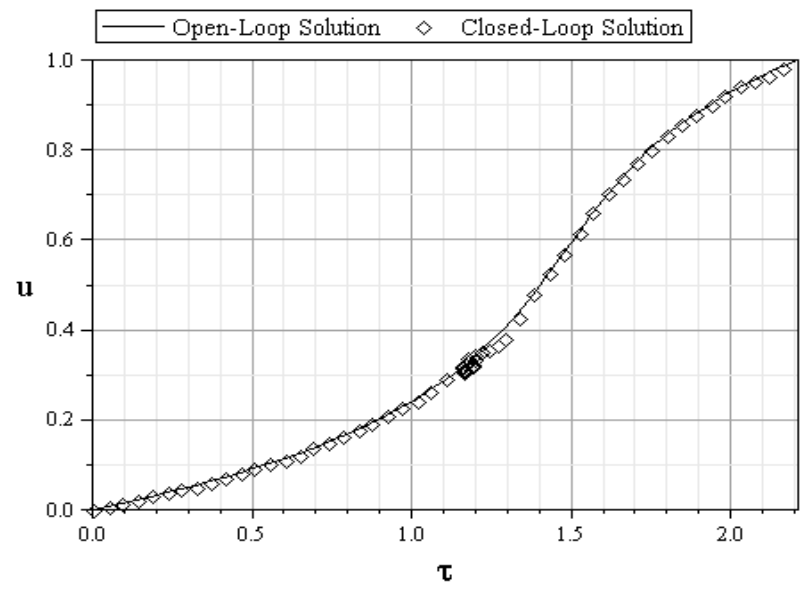

Fig. 6: Comparison between open-loop and closedloop solution of time-optimal horizontal velocity.

\section{CONCLUSION}

The determination of an analytical open-loop optimal control strategy for a nonlinear problem of satellite injection problem is achieved in this study. The open-loop results show that there is a good agreement between the investigated results and the analytical results of minimum-time feedback laws achieved for dynamic systems ${ }^{[3]}$. Then by, the closed-loop optimal control is computed using NOC law in the exact solution. This optimal closed-loop law can protect the satellite injection mission against the environment disturbances in each instant of time in the actual applications. Indeed, this performance is due to the fact that the NOC gains are functions of time. The comparison between the open-loop state-space trajectories with the closed-loop trajectories illustrates a good accuracy of NOC method for protecting the satellite mission on its nominal path. Another benefit to this approach is that it may be easily extend to dynamically varying parameters.

Considering the fact that the NOC law is a function of state variables perturbation, it can be easily illustrated that the instantaneous values of feedback gains $\left(K_{u}(\tau), K_{v}(\tau), K_{y}(\tau)\right)$ represent the sensitivity of the feedback law to the corresponding state variables perturbation namely $\delta u, \delta v$ and $\delta y$ respectively. Another advantage of the proposed strategy is that several difficulties associated with the numerical determination of optimal control solution for nonlinear systems such as slow convergence rate, undesirable singularity and high sensitivity to initial guesstimates are not appeared.

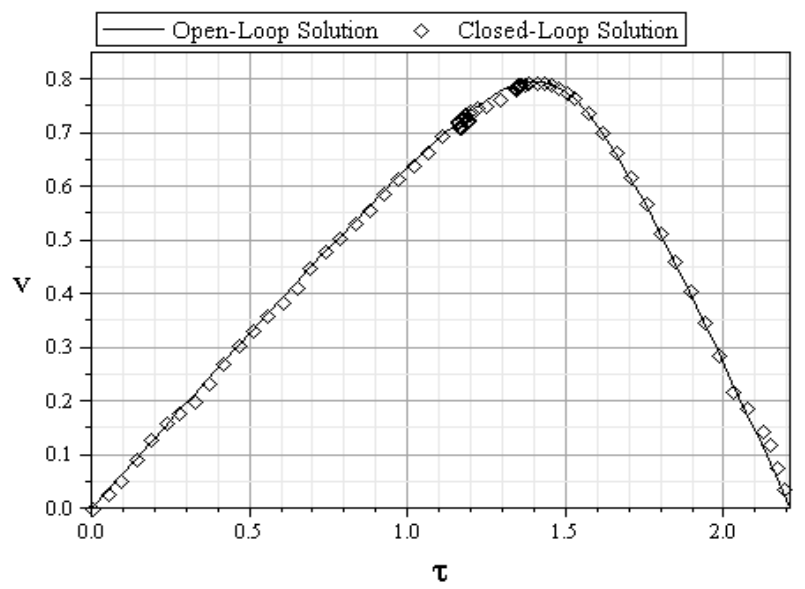

Fig. 7: Comparison between open-loop and closed-loop solution of time-optimal vertical velocity. 


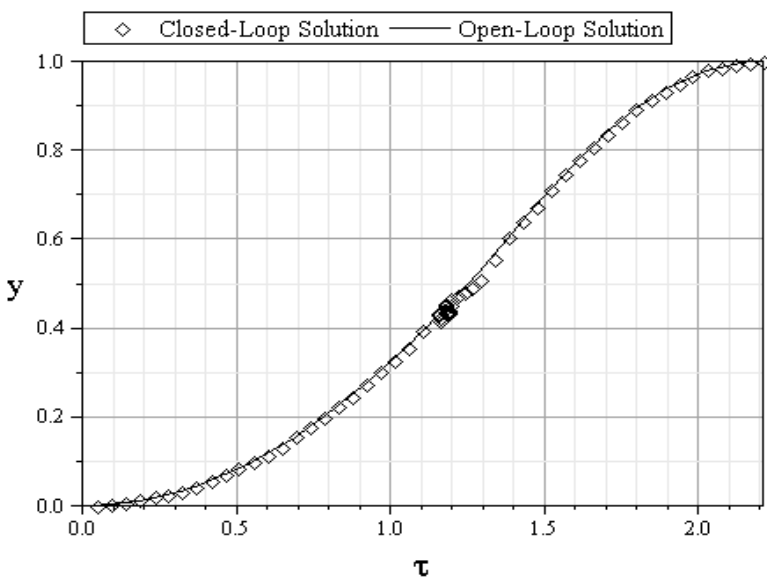

Fig. 8: Comparison between open-loop and closed-loop solution of time-optimal altitude.

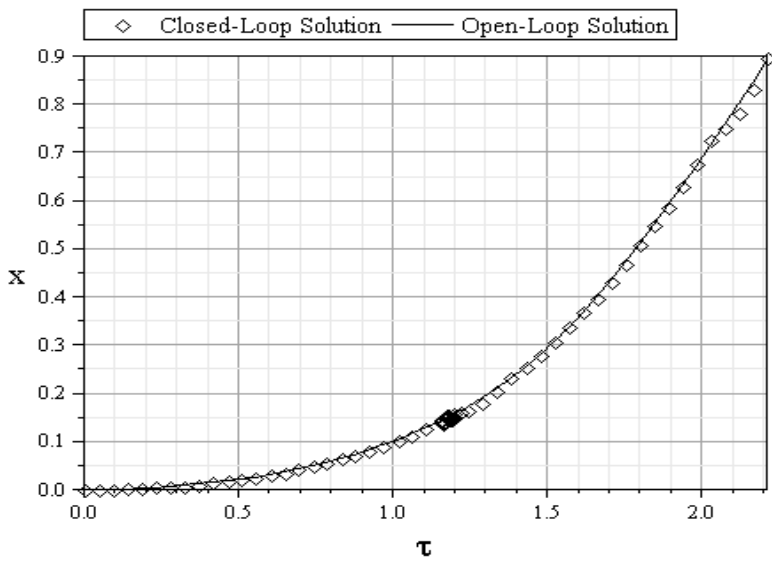

Fig. 9: Comparison between open-loop and closed-loop solution of time-optimal down range.

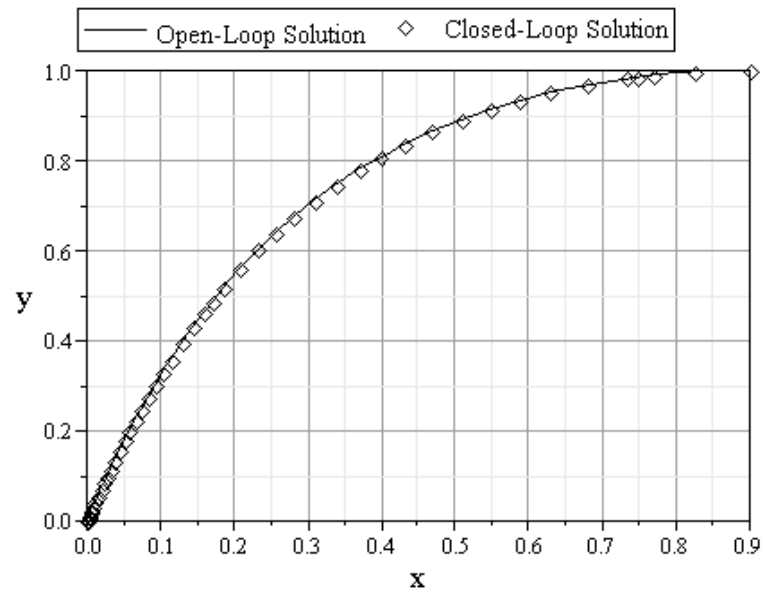

Fig. 10: Comparison between open-loop and closed-loop solution of time-optimal trajectory.

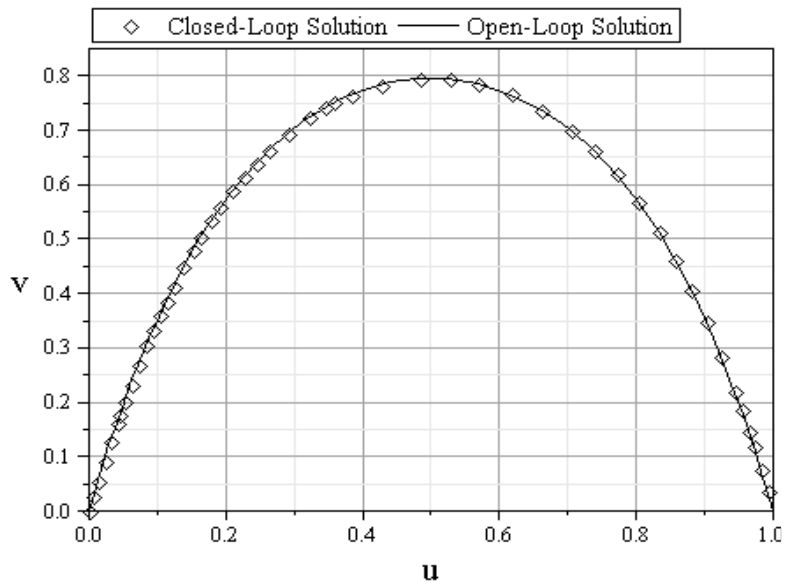

Fig. 11: Comparison between open-loop and closed-loop solution of time-optimal velocity.

\section{REFERENCES}

1. Bryson A.E., Ho Y.C., 1975. Applied Optimal Control: Optimization, Estimation, and Control, Hemisphere, New York, pp: 177 - 203.

2. Kirk D.E., 1970. Optimal Control Theory: An Introduction, Prentice-Hall, Engle wood Cliffs, New Jersey, pp: $202-209$.

3. Lee A.Y., Smyth P, 1993. Synthesis of Minimumtime Feedback Laws for Dynamics Systems Using Neural Networks. J GUID CONTROL DYNAM., 17 (4).

4. Jardin M.R., Bryson A.E, 2001. Neighboring Optimal Aircraft Guidance in Winds. J GUID CONTROL DYNAM., 24 (4): 710 -715.

5. Lee A.Y., Bryson A.E., 1989. Neighboring Extermals of Dynamic Optimization Problems with Parameter Variations. OPTIM CONTR APPL MET Journal, 10 (1): 39 - 52.

6. Pourtakdoust S.H., Novinzadeh A. B., Rahbar N., 2005. Time Optimal Closed-loop Fuzzy Control Strategy for Nonlinear Lunar Lander Mission, JAST, 2 (4): $27-36$.

7. Battin R.H., 2001. An Introduction to the Mathematics and Method of Astrodynamics, AIAA publication, Reston, pp: 550 - 567 .

8. Palma D., Magni L., 2005. On Optimality of Nonlinear Model Predictive Control, System and Control Letters journal, 56, pp: $58-61$.

9. Novinzadeh A.B., Pourtakdoust S. H., 2006. A Perturbation Approach in Determination of ClosedLoop Optimal-Fuzzy Control Policy for Lunar Landers, Japan Society for Aeronautical and Space Sciences, ISTS, pp: $134-141$. 
10. D’Souza C.N., 1997. An Optimal Guidance Law for Planetary Landing, AIAA Guidance, Navigation, and Control Conference, New Orleanse. Proc. LA, pp: pp: $11-16$.

11. Feeley T.S., Speyer J.L., 1994. Technique for Developing Approximate Optimal Advanced Launch System Guidance. J GUID CONTROL DYNAM, 17 (5), pp: 889 - 896.

12. Leung M.S., Calise A.J., 1994. Hybrid Approach to Near-Optimal Launch Vehicle Guidance. J GUID CONTROL DYNAM, 17 (5), 881 - 888.

13. Dabbous T.E., Ahmed N.U., 1982. Nonlinear Optimal Feedback Regulation of Satellite Angular Momenta. IEEE T AERO ELEC SYS, 18 (1), 2 10.

14. Nauidu D.S., Price D.B., 1987. Time Scale Synthesis of a Closed-Loop Discrete Optimal Control System. J GUID CONTROL DYNAM, $10(5), 417-421$.

15. Nauidu D.S., 2002. Singular Perturbation and Time Scales in Control Theory and Applications: An Overview. DYNAM CONT DIS SER B, 9, 233 78.
16. Bei C., Zhang W., 2008. A Guidance and Control Solution for Small Lunar Probe Precise Landing Mission, Acta Astronautica, 62(1), pp: $44-47$.

17. Suzuki S., Yoshizawa T., 1998. Near-Minimum Fuel Guidance Law of a Lunar Landing Module, IFAC Automatic Control in Aerospace, pp: 171 179.

18. Naghash A., Esmaelzadeh R., Mortazavi M., Jamilnia R., 2007. Near Optimal Guidance Law for Descent to a Point Using Inverse Problem Approach, AEROSP SCI TECHNOL Journal, 12 (3), pp: $241-247$.

19. Ardema M.D., 1976. Solution of the Minimum Time to Climb Problem by Matched Asymptotic Expansion, AIAA J, 14 (7), $843-850$. 